\title{
A multi-disciplinary design optimization of the passenger supply channel in the aircraft cabin
}

\author{
Mara Fuchs ${ }^{1, *}$, Christian Hesse ${ }^{1}$, Jörn Biedermann ${ }^{1}$, and Jörg Fuchte ${ }^{2}$ \\ ${ }^{1}$ DLR - German Aerospace Center, Institute of System Architectures in Aeronautics, Department \\ Cabin and Payload Systems, Hein-Saß-Weg 22, 21129 Hamburg, Germany \\ ${ }^{2}$ Diehl Aviation, Hein-Saß-Weg 22, 21129 Hamburg, Germany
}

\begin{abstract}
This paper shows the first results of a multidisciplinary design optimization (MDO) of the passenger service functions in the cabin. Therefore, both the placement of the components and the dimensions of the passenger supply channel (PSC) are varied. In order to modify the parameters, the individual components of the PSC are generated as objects using model based systems engineering. As a result, the information is stored in each object and can be used for the evaluation. In particular, the focus will be on passenger comfort and safety regulations. Collectively, this paper shows some possible variations for the arrangement of the PSC elements and discusses and compares the results obtained as well as possible future developments of this work.
\end{abstract}

\section{Introduction}

In recent years, the use of Model-based Systems Engineering (MBSE) and Multidisciplinary design optimization (MDO) for the design of complex products has increased. Especially in the aircraft design, an optimal solution of a system considering several domains such as maintenance, weight, cost, comfort or noise has to be found. A combination of both approaches enables an integrated view for the solution of complex systems.

In this paper, a MDO of the Passenger Supply Channel is performed. The PSC and the cabin itself are complex systems due to its many subsystems and their interactions. So, the model-based approach is used in the first step to describe the system and to establish a framework for a subsequent optimization. As a result, an evaluation of cabin concepts can already be carried out during the early development phase, whereby the requirements for passenger comfort and possible pre-assembly are in the foreground.

\section{Design Process of the Passenger Supply Channel}

The Passenger Supply Channel is an important aircraft cabin component and displays an interface between the crew, the aircraft and the passengers. It supports several service functions for the passenger. Some of them are important and necessary for a safe flight, for

* Corresponding author: mara.fuchs@dlr.de 
example the oxygen masks in case of a decrease in pressure, and some serve as a comfort aspect, for example the reading lights or the individual air conditioning. This results in different requirements for the design of the system and the placement of the individual functions.

In former times, the service functions were installed directly to the luggage racks. A quick adaptation to a new seat pitch or the removal of defective components was complicated and inflexible. The development of a Passenger Supply Channel changed that by decoupling the systems. Thus, the installation and removal of the service functions is independent. Moreover, the connections and supply of the systems can be laid through a central supply channel. However, these many cables and connections make the installation of the PSC elements very time-consuming and complex. Everything has to be installed manually and the configuration management for positioning the passenger services needs a lot of time. This results in high costs at the final assembly line for the original equipment manufacturer (OEM). One solution could be a pre-assembled Passenger Supply Channel to reduce time and costs. An automated installation processes for different seat layouts could improve the installation time and configuration management.

Another indication of a change in the PSC is the growing trend towards more accompanied luggage. During the last years, the airlines observe an increasing number of hand luggage in their aircraft cabins due to the rising costs for checked-in baggage [1]. The passengers' desire for more storage space in the cabin leads to new storage solutions or bigger overhead storage compartments (OHSC). Thus, the Passenger Supply Channel has to change as well and offers the potential for innovation and customization.

This paper describes the optimization of the Passenger Supply Channel regarding the passenger comfort and the assembly time. Section 2 gives a brief overview of Multidisciplinary design optimization and how the MBSE is used to describe the overall system and to create a central data base. Section 3 lists the requirements and boundaries of the optimization. Moreover it discusses the results and shows possible designs of the PSC that were obtained by the optimization. Section 4 gives a short outlook.

\subsection{The Passenger Supply Channel}

During the flight, the passenger has access to necessary service compartments in the cabin, for example the lavatories, as well as to their individual service functions. Latter are placed in the Passenger Supply Channel, which is built-in under the OHSC. The service functions are grouped into two categories. On the one hand, there are the primary functions, which are safety required [2]. These are the oxygen masks, the Fasten-Your-Seatbelt-Signs, the No-Smoking-Signs and the loudspeaker for the announcements. On the other hand, there are the secondary ones, which are provided by the airline as a service to the passenger. Examples would be the reading light, the attendant call and the individual air conditioning, which together are referred to as the Passenger Service. The various functions are shown exemplary in Figure 1 for an Airbus A320 cabin.

In general, the term Passenger Service Unit (PSU) is used to describe these several functions. But more precisely, the PSU is a panel that integrated the reading lights, the attendant call, the signs and the speaker [3]. Another important part of the PSU is the Passenger Interface and Supply Adapter (PISA). It controls and distributes the data to the PSU elements. Therefore, it is the interface between the cockpit, the cabin crew and the passenger and transmits the signals to the Cabin Intercommunication Data System (CIDS). In general, it is placed next to the reading lights and is not shown in the figure. 


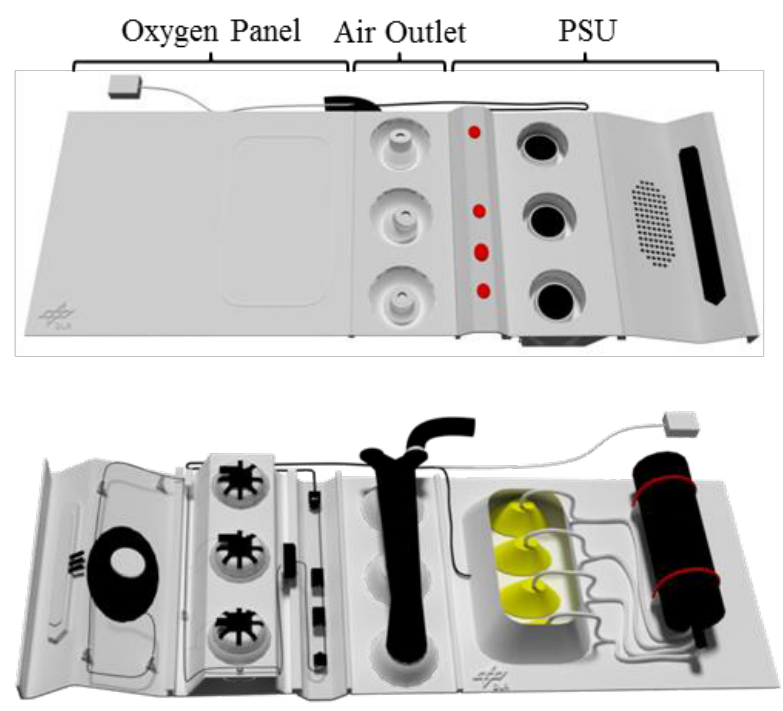

Fig. 1. The figure shows a typical design of the PSC elements of an Airbus A320 (according to [3]).

The arrangement and number of service functions vary with the passenger cabin configuration. Each seat row is assigned to one PSU. Filler panels between the units adapt the layout to the seat pitch. Although, the safety regulations and the passenger access to the primary functions must be ensured. Hence, the PSU and the other functions will be mounted to the Passenger Supply Channel during the final assembly. With its many interactions and subsystems, the PSC is appropriated for an initial implementation of the model-based approach to aircraft cabin design and subsequent for a multi-disciplinary design optimization.

\subsection{Implementation of rules and knowledge (MBSE)}

As mentioned before, there are various interactions between the PSC and other cabin systems as well as an interface to the passenger. Therefore, the model-based approach is chosen for the description of the overall system. The model-based systems engineering enables the methodical development of a system. It focuses a holistic understanding of the requirements and the elaboration of solutions [4]. In addition, complex interactions between the subsystems are better visualized using models [4].

The different models used in this study are shown below [Fig. 2]. The geometry model is created for the visualization of the cabin structure and the structure of the PSC. In addition, this provides the boundary conditions for the placement of the service functions by specifying the geometric dimensions of the Passenger Supply Channel. Expanding on this data, the ontology of the entire system is derived in another model to describe the hierarchy of the components. Moreover, a requirement model is used to define the objectives and specifications for the placement of the PSC elements. As a result, different designs are studied because the model refers to varying rules within the knowledge database based on the defined objectives. A functional model is needed for representing interfaces to other subsystems of the cabin or the passenger. In order to evaluate the placement of the passenger service functions, two more models are needed. First, an assembly cost model is created to record potential savings in pre-assembly. Second, a passenger model is used for the consideration of comfort in the cabin. A further insight into the structure of the models and the architecture can be seen in [3]. 


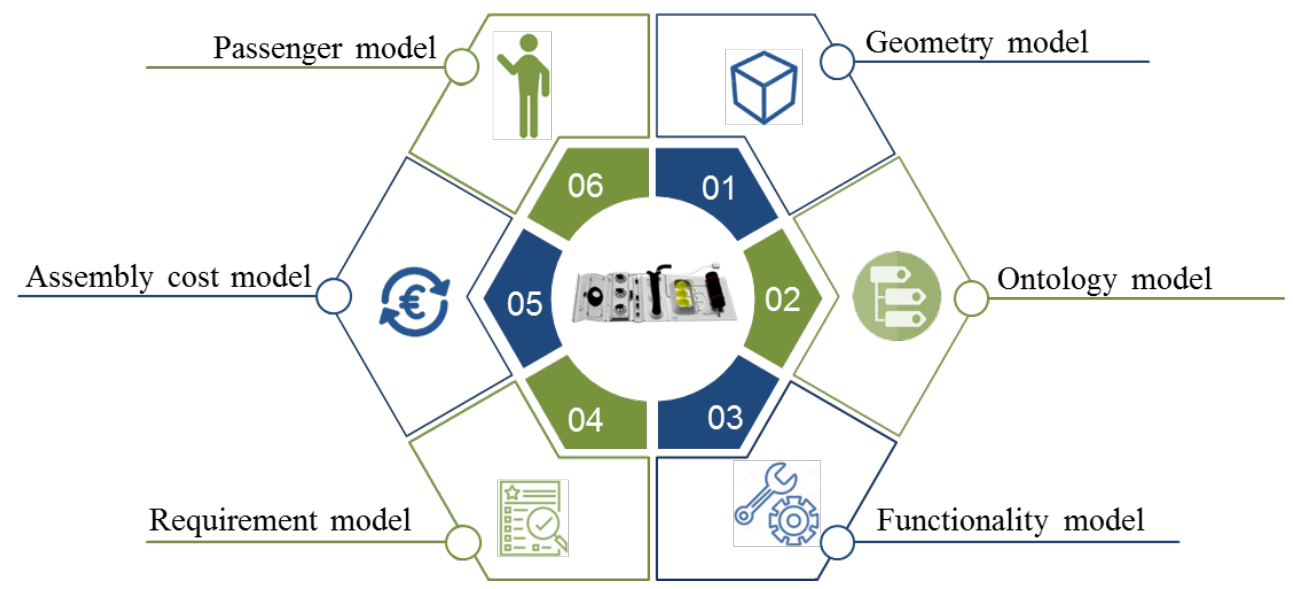

Fig. 2. The six generated models to describe the System PSC.

In a next step, these models are used to optimize the design of the Passenger Supply Channel, taking into account the passenger comfort and cost savings due to a pre-assembly.

\subsection{Multi-disciplinary design optimization}

The Multi-disciplinary design optimization uses methods to maximize or minimize an objective function incorporating a number of disciplines while satisfying determined constraints [5]. Therefore, the optimization problem is defined by the objective function, the design variables and the constraints [5]. The objective function enables a comparison of two or more designs. So, it has to be a numerical value that can be minimized or maximized to measure the quality of a design. Especially for the Passenger Supply Channel, the two objectives are maximizing the passenger comfort and minimizing the assembly costs.

In addition, the design variables are to parameterize the observed system and are varied during the design process. So, the algorithm of the optimization is choosing the design values that yield an optimum design by satisfying the objective functions [5]. The number of design variables can expand, but the examined ones in this paper are the length of the oxygen panel, the width of the supply channel and the number of channels.

At last, the constraints are set to border the design variables. It can reflect user requirements or physical laws [5]. In this context, the design variables are limited by the technically possible geometrical sizes of the cabin components and the standard size of today`s PSC.

Due to the comparison of two objectives, we use a method that allows a multi-objective optimization, such as the calculation of a Pareto front. The Pareto front shows the optimal solutions for the examined problem. That means it is not possible to improve an objective without degrading the others [6]. All solutions off the front are dominated by the ones on the Pareto front and thus are not efficient. Therefore, the optimal solution can be found in the Pareto front.

\section{MDO of the Passenger Supply Channel}

The following section describes the optimization process and the considered assumptions. Furthermore, the results of the multi-disciplinary design optimization are shown and discussed. The focus is on a cabin for a single-aisle aircraft. 


\subsection{Optimization}

In this study, both the models and the rules are programmed using Matlab R2018b. First, a script calls up the stored rules after the other and generates the individual models taking into account the requirements. For optimization, the following three design values are examined: the length of the oxygen panel, the width of the Passenger Supply Channel and the number of channels. The boundary conditions of these were set to the listed values, wherein the smallest value is chosen so that an implementation can be ensured (Table 1). Moreover, the maximum value is set to the current standard of the Airbus A320 as a reference. So, the potential by reducing the components geometry are investigated. In addition to the design variables, two different overhead storage compartments are studied. The first one is based on today's OHSC for a single aisle aircraft. It is characterized by a central channel, which allows every passenger in a row the same access. In the following, it is called standard bin (STD). In contrast, there is an enlarged bin (ENL), which offers more storage space for the passenger in the cabin. The position of the supply channel changes and moves towards the window seat. As a result, the installation of another supply channel is possible.

Table 1. Design values and their boundaries.

\begin{tabular}{|c|c|c|}
\hline Design value & Lower bound & Upper bound \\
\hline $\begin{array}{c}\text { Length of the } \\
\text { oxygen panel }\end{array}$ & 5 inch & 12 inch \\
\hline Width of the PSC & 5 inch & 11 inch \\
\hline $\begin{array}{c}\text { Number of } \\
\text { Channels }\end{array}$ & 1 & 2 \\
\hline
\end{tabular}

The two compared objectives are the comfort of the passenger $\left(f_{1}\right)$ and the time/cost of the assembly $\left(f_{2}\right)$. Equations (1) to (2) show the calculation of the distance $\mathrm{a}_{\text {tat, } \mathrm{i}}$ between the reference points of the passenger and the individual reference point of each service function. Comparing this value to a limit value $a_{\text {max,i }}$ gives a statement about the passenger comfort. Subsequently, all distance values $Z_{\mathrm{i}}$ are accumulated and divided by six to obtain an average. A smaller value of $f_{1}$ means, that the average European can use the service functions accordingly while a higher value (close to one) reaches the limit and therefore is no longer comfortable.

$$
\begin{gathered}
Z_{\mathrm{i}}=\frac{\sum a_{\mathrm{tat}, \mathrm{i}}}{n_{\mathrm{S}}} \\
a_{\mathrm{max}, \mathrm{i}} \\
f_{1}=\frac{\sum Z_{\mathrm{i}}}{6}
\end{gathered}
$$

Equation (3) shows the calculation of the assembly time. P describes the number of the PSUs, $t_{i}$ the installation time of each element and $\mathrm{N}$ is the Non-Value Adding, which differentiated between a pre- and final assembly. The second part of the equation considered the installation time of the filler panels $t_{\mathrm{f}}$. The number of filler panels $\mathrm{F}_{\mathrm{P}}$ varies with the length of the PSU. Concluding, dividing the values by 100 converts it into the unit [h]. The optimal solutions of the defined problem will be found by minimizing the two objectives $\left(f_{1}, f_{2}\right)$.

$$
f_{2}=P \cdot\left(\sum_{i}^{n} t_{\mathrm{i}}\right) \cdot \frac{(1+N)}{100}+\frac{t_{f} \cdot F_{P}}{100}
$$


We used the optimization toolbox of the PlatEMO v1.6 and the multi-objective evolutionary algorithm (MOEA) NSGA-II to minimize the objective functions and to create the Pareto front [7]. The algorithm maintains a set of individuals that altogether are called a population. Every individual represents a solution. During the evolution, the population is updated in each generation and new individuals are generated. The evolutionary process stops when the population approximates the Pareto front [7]. The parameters used for the optimization are population size $=10$, mutation rate $=0.1$ and number of evaluations $=80$.

\subsection{Optimization results for the PSC}

The following graph shows the results for the multi-objective design optimization of the Passenger Supply Channel by varying the width of the PSC and the length of the oxygen panel (Fig. 4). The value for the passenger comfort is shown on the $\mathrm{x}$-axis and the assembly time is shown on the y-axis. The round circles display the results of the individual solutions. In contrast, the marked circles show the Pareto front of the last epoch during the optimization. The result for today`s design is not shown in the figure.

Figure 4 shows a gap between the points, forming two areas. This leads to a step in the results for the passenger comfort. This is due to the dimensions of the oxygen panel. For a pre-assembled design, the limits of the overhead storage compartments must be adhered to. From a certain length of the oxygen panel, the arrangements of the other PSC-elements and the potential installation space is restricted due to the higher seat pitch at the emergency exits. This changes the accessibility of the elements for the passenger and the comfort value deteriorates. Therefore, the length of the oxygen panel is limited to 9.5 inch for a preassembled version.

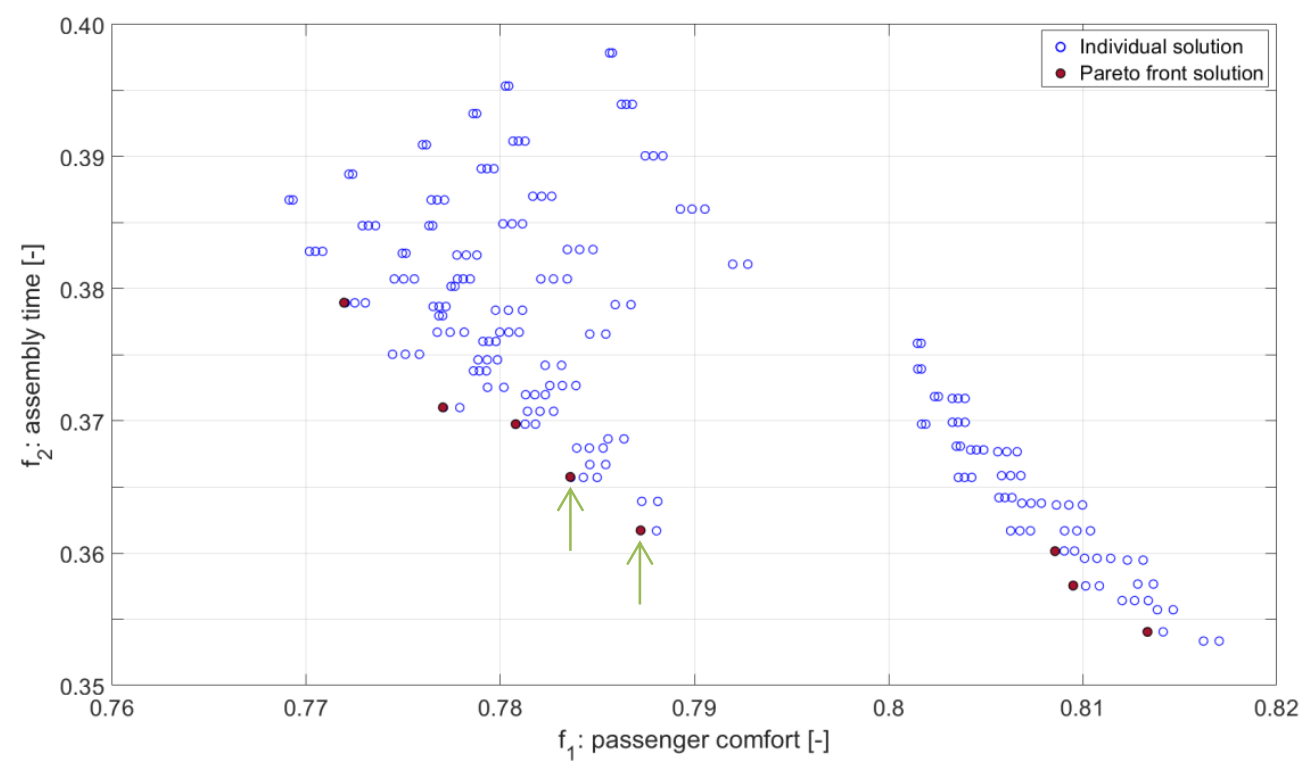

Fig. 4. The graph shows the results of the multi-objective design optimization for the two objective functions (scenario: $1 \mathrm{PSC}$, pre-assembly).

The calculation of the objective function for the passenger comfort is based on the summation of the several evaluation criteria and distances for the PSC elements. In order to exclude dominance by one of the comfort functions, the optimization process was also done for only one of the service elements. Various studies showed no dominance by any of the 
evaluation equations. The results were similar and show the same trend for the progression of the Pareto front.

Based on the results, further findings can be derived. First, a smaller oxygen panel achieves lower values for the comfort target function due to the arrangement of the other service functions in the channel. Second, a bigger panel affects the assembly time, because the number of filler panels is reduced. Third, the design of the PSC and its elements is similar for both the standard bin and the enlarged bin. Moreover, a compact design is more suitable for a pre-assembled variant. Nevertheless, good values for the passenger comfort can be achieved. Fourth, the dimension of the today`s standard oxygen panel of an Airbus A320 is not optimal for a pre-assembled version.

Finally, an optimal design for the PSC can be found. If we compare every value of the Pareto front solutions, we can see that there is not the one optimal solution of a design with respect of the assembly time and costs. There are several other combinations with, for example, a better value for the assembly costs with deterioration of the passenger comfort value. Therefore, a compromise between low assembly time and high comfort is recommended. The best design will then be achieved when the oxygen panel has a length of 9.5 inch and the Passenger Supply Channel has a width of 9.5 to 11 inch (marked arrows). For this configuration, the value of $f_{1}=0.78$ and for $f_{2}=0.36$. Comparing these values to the ones of today's PSC design $\left(f_{1}=0.74, f_{2}=1.0\right)$, we can obtain a reduction in assembly time without losing major comfort.

Figure 5a shows one of the designs of a pre-assembly Passenger Supply Channel for the ENL with the service functions for three seat rows. The several service functions are shown exemplary. This example is generated with a PSC width of 10 inches and an oxygen box length of 9.5 inch. The design is suitable for an installation as well in a standard bin as in an enlarged bin. In addition, the service functions can be arranged either symmetrically for a unitary setup or alternately to ensure the requirements of a pre-assemble design. For both cases, this results in good values for the passenger comfort. Figure $5 \mathrm{~b}$ shows a similar design of the PSC but with two supply channels. In comparison to the other design, the comfort can be improved due to the distribution of the service functions $\left(f_{1}=0.68\right)$. However, the installation time increases because of the additional filler panels in the second $\operatorname{PSC}\left(f_{2}=0.69\right)$.
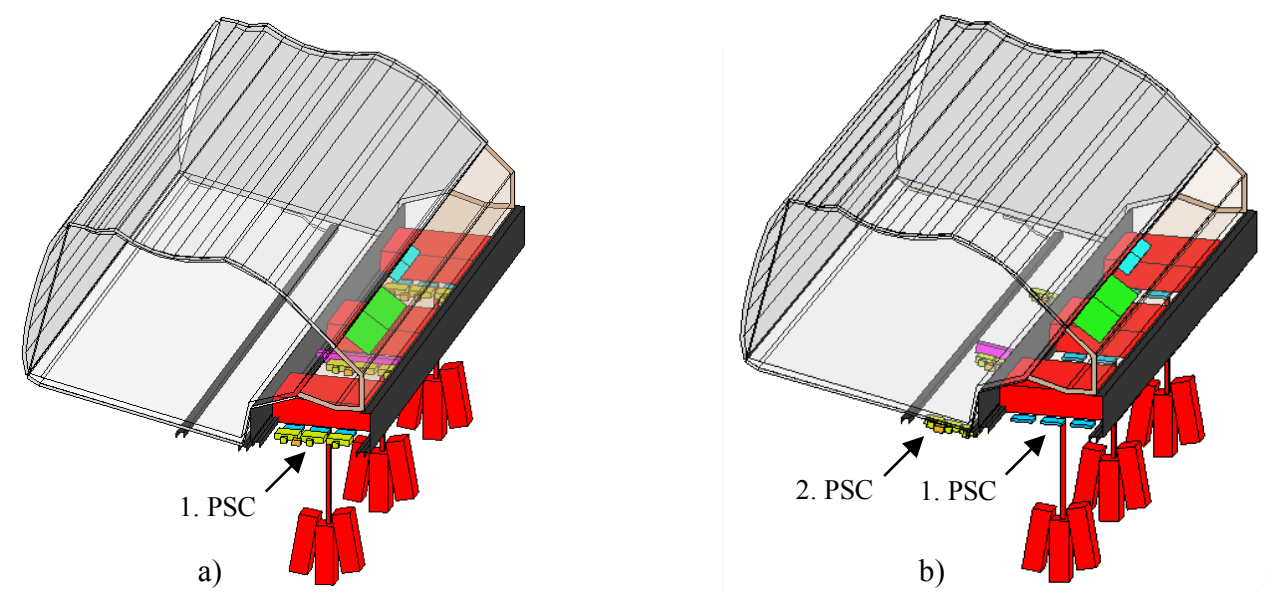

Fig. 5. The left picture shows the recommended design of the PSC and its elements. The picture on the right shows the similar design with a distribution of the service functions on two Passenger Supply Channels (oxygen panel: 9.5 inch, PSC width: 10 inch, ENL). 
The compact arrangement of the service functions within the OHSC borders creates a module. As shown in Figure 5, the modules can be produced and mounted by one manufacturer (Tier 1) and then delivered to the original equipment manufacturer for the final assembly into the aircraft cabin. Thereby, the interfaces and connections during the installation can be reduced due to the potential for a plug and play solution.

\subsection{Constraints of the optimization}

The assumptions made enables first good results for an optimization of the Passenger Supply Channel. The specified parameter limits of the design values, however, require the scope for optimization. So, the results considered could vary even further. Besides, the designs were not fully checked for technical feasibility. This may require an adjustment of the results and possibly leads to a different optimal variant. Nevertheless, this work provided a first impression of the optimization potential of the Passenger Supply Channel.

\section{Outlook}

The results show, that a different arrangement of the passenger service functions reduces the time of assembly and therefore reduces installation costs due to pre-assembled service functions. In addition, the passenger comfort is thereby changed only slightly so that the airline can ensure the same comfort, taking into account the safety requirements. Therefore, an enlarged overhead storage compartment with two Passenger Supply Channels is the optimal design for this case. In a next step, this approach can be extended to the design of further cabin systems. Moreover, the evaluation criteria can be improved and more objective functions developed.

\section{References}

1. C. McGuire, Stuffing your hand luggage into the overhead cabin on planes is about to get A LOT more difficult, https://www.thesun.co.uk/travel/4102033/stuffing-yourhand-luggage-into-the-overhead-cabin-on-planes-is-about-to-get-a-lot-more-difficult/ The Sun (2017), (accessed 25. July 2019)

2. European Aviation Safety Agency, Certification Specifications for Large Aeroplanes CS-25, Amendment 3, 2007

3. M. Fuchs, J. Fuchte, J. Biedermann, Ein MBSE-Ansatz für die Auslegung von Flugzeugkabinen am Beispiel eines Passenger Supply Channel, DLRK (2019)

4. SE INCOSE, Vision 2020 (INCOSE-TP-2004-004-02), 2007

5. A. C. Marta, Multidisciplinary Design Optimization of Aircrafts, Técnico Lisboa, lecture series (2015)

6. Cenaero, Software Pareto Front, http://www.cenaero.be/Page.asp?docid=27103\& (accessed 23. July 2019)

7. Y. Tian, R. Cheng, X. Zhang, Y. Jin, PlatEMO: A Matlab Platform for Evolutionary Multi-Objective Optimization, Educational Forum, IEEE Computational Intelligence Magazine, 12(4): 73-87 (2017) 